\title{
Um estudo de caso da remoção da palhada de cana-de-açúcar do solo: quais são as implicações no conteúdo de matéria orgânica do solo?
}

\author{
A case study of sugarcane straw removal from the soil: what are the implications for soil organic \\ matter content?
}

Un estudio de caso de remoción de paja de caña de azúcar del suelo: ¿cuáles son las implicaciones para el contenido de materia orgánica del suelo?

\author{
Rakiely Martins da Silva \\ ORCID: https://orcid.org/0000-0001-6914-4721 \\ Universidade Estadual do Norte Fluminense, Brasil \\ E-mail: rakiely@ hotmail.com.br \\ Rafaela Martins da Silva \\ ORCID: https://orcid.org/0000-0002-7733-8208 \\ Universidade Federal de Viçosa, Brasil \\ E-mail: rfeng.florestal@gmail.com \\ Jheny Kesley Mazzini de Souza \\ ORCID: https://orcid.org/0000-0001-8188-4423 \\ Universidade Federal de São João Del Rei, Brasil \\ E-mail: Jheny.kesley@hotmail.com \\ Jianne Rafaela Mazzini de Souza \\ ORCID: https://orcid.org/0000-0002-0803-4438 \\ Universidade Federal de Viçosa, Brasil \\ E-mail: jianne.rafaela@hotmail.com \\ Tatiane Pimentel Gedijesk \\ ORCID: https://orcid.org/0000-0002-3382-9283 \\ Universidade Federal Rural do Rio de Janeiro, Brasil \\ E-mail: tatianepgagro@gmail.com
}

\begin{abstract}
Resumo
O Brasil é o maior produtor de cana-de-açúcar do mundo. Com as mudanças nos sistemas de colheita da cana-deaçúcar, há uma quantidade expressiva de palhada que fica depositada sobre o solo, podendo contribuir para o aumento da matéria orgânica do solo (MOS). No entanto, no Brasil já existem diversas Unidades Industriais que processam toda a biomassa da cana para fabricar álcool de segunda geração e/ou gerar energia elétrica, tornando-se assim, uma nova fonte de renda das Usinas e Destilarias. Ao processar toda a biomassa gerada pela cana-de-açúcar, os possíveis efeitos favoráveis sobre as propriedades químicas, físicas e biológicas do solo serão retardados. Vale destacar que a matéria orgânica determina os principais atributos que definem a qualidade do solo, sendo diretamente afetada pelas práticas de manejo. Por meio do estudo da qualidade da matéria orgânica, pode-se além de avaliar a qualidade do solo, ter uma maior compreensão do sistema de produção que este solo comporta. Diante do exposto, o objetivo do presente trabalho, foi de estudar os principais efeitos da retirada da palhada da superfície dos solos no conteúdo de matéria orgânica do solo, indicando estudos que apontam a quantidade ideal de palhada a ser deixada no solo, sem afetar o conteudo de matéria orgânica. Para isto, foi realizado uma intensa busca em bases de dados, de estudos que avaliaram as possíveis implicações da retirada total e parcial da palhada de cana.
\end{abstract}

Palavras-chave: Saccharum officinarum; Sustentabilidade; Qualidade do solo.

\section{Abstract}

Brazil is the largest sugarcane producer in the world. With the changes in sugarcane harvesting systems, there is an expressive amount of straw that is deposited on the soil, which can contribute to the increase of soil organic matter (MOS). However, in Brazil there are already several Industrial Units that process all the sugarcane biomass to produce second generation alcohol and/or generate electricity, thus becoming a new source of income for the Plants and Distilleries. By processing all the biomass generated by sugarcane, the possible favorable effects on the chemical, physical and biological properties of the soil will be delayed. It is worth noting that organic matter determines the main attributes that define soil quality, being directly affected by management practices. Through the study of the quality of organic matter, it is possible, in addition to evaluating the quality of the soil, to have a greater understanding of the production system that this soil comprises. Given the above, the objective of this work was to study the main effects of removing straw from the soil surface on the organic matter content of the soil, indicating studies that 
indicate the ideal amount of straw to be left in the soil, without affecting the soil. organic matter content. For this, an intense search was carried out in databases, for studies that evaluated the possible implications of the total and partial removal of cane straw.

Keywords: Saccharum officinarum; Sustainability; Soil quality.

\section{Resumen}

Brasil es el mayor productor de caña de azúcar del mundo. Con los cambios en los sistemas de cosecha de la caña de azúcar, hay una cantidad expresiva de paja que se deposita en el suelo, lo que puede contribuir al aumento de la materia orgánica del suelo (MOS). Sin embargo, en Brasil ya existen varias Unidades Industriales que procesan toda la biomasa de la caña de azúcar para producir alcohol de segunda generación y / o generar electricidad, convirtiéndose así en una nueva fuente de ingresos para las Plantas y Destilerías. Al procesar toda la biomasa generada por la caña de azúcar, se retrasarán los posibles efectos favorables sobre las propiedades químicas, físicas y biológicas del suelo. Cabe señalar que la materia orgánica determina los principales atributos que definen la calidad del suelo, siendo directamente afectada por las prácticas de manejo. A través del estudio de la calidad de la materia orgánica, es posible, además de evaluar la calidad del suelo, tener un mayor conocimiento del sistema de producción que comprende este suelo. Dado lo anterior, el objetivo de este trabajo fue estudiar los principales efectos de la remoción de paja de la superficie del suelo sobre el contenido de materia orgánica del suelo, indicando estudios que indican la cantidad ideal de paja a dejar en el suelo, sin afectar la contenido de materia orgánica del suelo. Para ello, se realizó una búsqueda intensa en bases de datos, de estudios que evaluaran las posibles implicaciones de la remoción total y parcial de la paja de caña.

Palabras clave: Saccharum officinarum; Sustentabilidad; Calidad del suelo.

\section{Introdução}

O aumento da população mundial tem sido acompanhado pela crescente demanda por alimentos e, em paralelo, a alarmante necessidade de novas áreas agrícolas e recursos naturais. Nesse sentido, surgiram preocupações com a sustentabilidade dos sistemas agrícolas (FAO, 2015). A produção de cana-de-açúcar é de suma importância no cenário econômico brasileiro, sendo o Brasil o maior produtor e exportador mundial da cultura (FAO, 2020). A cultura é considerada uma das grandes alternativas para o setor de biocombustíveis devido ao grande potencial na produção de etanol e aos respectivos subprodutos. Além da produção de etanol e açúcar, as unidades de produção têm buscado operar com maior eficiência, inclusive com geração de energia elétrica, auxiliando na redução dos custos e contribuindo para a sustentabilidade da atividade.

O grande desafio da produção de cana-de-açúcar é buscar sistemas de colheita que proporcionem altos rendimentos e que não aumentem os danos ambientais. Desta forma, a abolição da queima na colheita dos canaviais, de forma gradativa nas regiões produtoras, tem se mostrado eficiente em termos de rendimento de trabalho, qualidade do ar e do solo.

A queima da palhada, realizada antes da colheita manual, é uma forma de manejo ainda utilizada na cultura da canade-açúcar em algumas regiões produtoras, com a premissa de eliminar resíduos vegetais e animais peçonhentos, facilitando o trabalho dos cortadores e do transporte dos toletes para a usina, além de provocar uma queda na população de importantes pragas da cultura (Arrigoni, 2013; Macedo \& Macedo, 2004). Entretanto, esta prática pode ocasionar danos nocivos à saúde humana, além de um aumento considerável na emissão de gases poluidores e causadores do efeito estufa (Carvalho et al., 2017). Por outro lado, a colheita da cana crua (mecanizada) sem queima prévia, conserva uma cobertura de resíduo vegetal (palhada) sobre o solo, contribuindo para o potencial produtivo da cana-de-açúcar. No sistema com colheita sem queima da palha são adicionados de $10 \mathrm{a} 20 \mathrm{Mg} \mathrm{ha}^{-1}$ ano $^{-1}$ de matéria seca sobre o solo, e têm-se um aporte de $\mathrm{C}$ anual que varia de 4,7 a 9,4 $\mathrm{Mg} \mathrm{ha}^{-1}$ (Schultz et al., 2010).

A palhada da cana acumulada na superfície do solo apresenta baixa taxa de mineralização, podendo variar de acordo com o clima e a textura do solo. No entanto, pode-se destacar como benefícios, em longo prazo, a manutenção da fauna do solo, o aumento do teor de matéria orgânica no solo, a menor perda de umidade e menor susceptibilidade à erosão (Souza et al., 2005). Por conseguinte, a manutenção da palhada promove alterações nos estoques de carbono e nitrogênio no solo, afetando principalmente, a dinâmica do carbono e nitrogênio, a humificação da matéria orgânica (Pinheiro et al., 2010). 
As diferentes frações de carbono orgânico humificado extraído do solo possuem características químicas, físicas e morfológicas diferenciadas e a distribuição destas frações no solo pode indicar, portanto a qualidade da matéria orgânica presente (Canellas \& Santos, 2005). O monitoramento da qualidade do solo em função de práticas de manejo da palhada da cana-de-açúcar na colheita é fundamental para o desenvolvimento sustentável do sistema de produção desta lavoura. O objetivo do trabalho é de relatar sobre os sistemas de colheita de cana-de-açúçar (Colheita sem queima e com queima) e o que esses diferentes sistemas de colheitas influenciam na qualidade da matéria orgânica do solo.

\section{Metodologia}

A presente pesquisa trata-se de um estudo de caso único sistemático, de caráter exploratório, caracterizado por ser uma pesquisa qualitativa (Pereira, 2018). O estudo de caso é utilizado quando existe a intenção de investigar uma realidade, compreendendo-a de forma integral, descritiva e interpretativa, em busca pela descoberta, trazendo como resultado a construção de conhecimento através da escrita científica (Pereira, 2018; Coimbra \& Martins, 2013). A pesquisa exploratória, é aquela que visa proporcionar uma maior familiaridade com o problema de um tema, ainda pouco conhecido (Gil, 2008). A obtenção dos dados e a interpretação dos resultados são os focos principais da abordagem, dependendo da capacidade e estilo do pesquisador (Gil, 2002).

O levantamento de dados, ocorreu após a observação da lacuna entre a quantidade ideal de palhada de cana-de-açúcar a ser deixada no solo, sem que esta venha interferir no conteúdo de matéria orgânica em solos de área canavieira, afetando dessa forma, a qualidade do solo. Para tal estudo, foi realizado uma vasta procura por estudos que relatam a representatividade do setor sulcroalcooleiro, passando pela significância da implementação de novas tecnologias como a mecanização do sistema de colheita, até os impactos dessa atividade na qualidade do solo, sobretudo na qualidade da matéria orgânica do solo, que se relaciona direta e indiretamente a diversos fatores na interface solo-planta. As palavras-chave cana-de-açúcar, manejo de palhada, matéria orgânica, substâncias húmicas, sustentabilidade e suas associações foram usadas como termos de pesquisa no banco de dados ISI Web of Science,Google Scholare Scopus, sendo selecionados apenas 22 estudos, que continham informações suficientes para serem incluídos em nosso estudo. Incluímos artigos que apresentavam informações sobre a geração de energias sustentáveis, expansão e evolução da cana-de açúcar no Brasil, séries históricas da safra brasileira de canade-açúcar, indicadores de qualidade ambiental, manejo e conservação do solo e indicadores edafoclimáticos. Os estudos foram compilados e analisados, buscando a compreensão de como as estratégias e práticas adotadas no cultivo de cana-de-açúcar influenciam na qualidade e no conteúdo da matéria orgânica do solo, um processo fundamental para garantir a sustentabilidade do sistema de produção.

\section{Resultados e Discussão}

\subsection{Sistemas de colheita da cana-de-açúcar no Brasil}

Atualmente, o Brasil é o maior produtor mundial de cana-de-açúcar (747 milhões de toneladas em 2020), além disso, Índia (377), China (108), Tailândia (104) e Paquistão (67) também se destacam entre os cinco maiores produtores de cana (milhões de toneladas) em ordem decrescente (FAO, 2020). O aumento da demanda mundial por etanol, oriundo de fontes renováveis, aliado à grandes áreas cultiváveis e condições edafoclimáticas tornam a cana-de-açúcar uma cultura de destaque no cenário nacional e internacional, com grande importância econômica.

Há dois diferentes sistemas de colheita de cana-de-açúcar: a) sistema de queima da cana-de-açúcar e b) sistema de colheita mecanizada sem queima e deposição de palha na superfície do solo. O sistema de queima é resultante do avanço no setor sucroalcooleiro, nos anos 50, que estimulou práticas que facilitassem o manejo da cultura, a sua colheita e ainda reduzissem a mão-de-obra (Cardoso et al., 2019). Com isso, a queima prévia do canavial, passou a ser bastante difundida nas 
áreas canavieira.

A colheita queimada consiste, na utilização do fogo, como uma ferramenta, cujo objetivo é eliminar o excesso de resíduos vegetais presentes na superfície do solo, além de diminuir a população de animais peçonhetos, facilitando o trabalho dos cortadores e do transporte dos toletes para a usina (Cardoso et al., 2019; Du et al., 2018). No entanto, a queima da palhada contribui para a emissão de dióxido de carbono (CO2), monóxido de carbono (CO), metano (CH4), óxido nitroso (N2O), óxidos de nitrogênio (NOx) e partículas da queima das plantações tem importantes impactos ambientais e sociais, aumentando o risco de doenças respiratórias e outros problemas de saúde em trabalhadores de campo e na população vizinha (Lopes et al., 2017; Paraiso \& Gouveia, 2015).

A prática da queima antes da colheita também está associada ao manejo de pragas na lavoura. A queimada, provoca uma queda na população da broca-da-cana (Diatrea saccharalis), na cigarrinha-da-índia (Mahanarva fimbriolata) e sphenophorus (Sphenophorus levis), sendo, portanto, um modo de controle da praga da cultura, trazendo aumentos significativos no rendimento da cultura (Arrigoni, 2013; Dinardo-Miranda \& Fracasso, 2013) e na qualidade da matéria prima (Rossato et al., 2013). Vale ressaltar que embora a remoção da palhada apresente redução na população de cigarrinhas-dasraízes, o nível de controle populacional não permite que outras práticas de controle sejam descartadas, como métodos químicos e / ou biológicos (Castro et al., 2019). Por outro lado, quando a palhada é deixada sobre o solo, no sistema de colheita mecanizada sem a queima, outras pragas importantes da cultura da cana-de-açúcar, como os nematoides parasitas e besouros, como o Migdolus fryanus, não foram afetadas (Carvalho et al., 2017; Dinardo-Miranda \& Fracasso, 2013).

A queima da cana-de-açúcar tem efeitos negativos na qualidade do solo. Com essa prática, menos serapilheira é depositada no solo, diminuindo a ciclagem de nutrientes, a estabilidade de agregados e, consequentemente, o fluxo subsuperficial (Vasconcelos et al., 2014). Além disso, remove os resíduos orgânicos da superfície do solo, reduzindo o teor de matéria orgânica (Pinheiro et al., 2010).

Nas últimas décadas, políticas governamentais e iniciativas público-privadas têm dado suporte adequado para a adoção de canaviais sem queima, associados a menor impacto ambiental, conservação do solo e proteção à saúde humana (Silva et al., 2021). No Estado de São Paulo, o maior produtor de cana-de-açúcar do país (IBGE 2020), a Lei Estadual $\mathrm{n}^{\circ}$.11.241/02 estabeleceu que o uso do fogo como método despalhador e facilitador do corte da cana deveria ser abolido de forma gradativa, sendo totalmente eliminado até $2021 \mathrm{em}$ áreas com declive do terreno de até 12\% (Carvalho et al., 2017). Dessa forma, a colheita mecanizada da cana-de-açúcar está cada vez mais presente nos sistemas de produção no Brasil. Cerca de $94 \%$ dos canaviais no centro-sul do Brasil estão sendo colhidos mecanicamente sem queima da palha no que é chamado de sistema de cana verde (Bordonal et al., 2018).

Estudos realizados com cana-de-açúcar no Brasil mostram que a queima da cana-de-açúcar por mais que 5 anos consecutivos pode reduzir a quantidade de fósforo orgânico (P) ligado aos ácidos húmicos, principalmente diéster ortofosfato e fosfatos de açúcar (Rossi et al., 2013). Também foi demonstrado que reduz o conteúdo de carbono orgânico total (COT) do solo em até 50\% (Souza et al., 2012) e modifica as propriedades estruturais dos ácidos húmicos, aumentando a presença de compostos aromáticos (Rossi et al., 2016).

No sistema de colheita mecanizada, isso é, sem o uso do fogo, os restos vegetais (folhas, bainhas, ponteiros, pedaços de colmo) são cortados, triturados e lançados sobre a superfície do solo formando uma cobertura de resíduo vegetal denominada palha ou palhada (Menandro et al., 2017). A quantidade de palhada depositada nos solos canaviais colhidos sem queima varia de 10 a $20 \mathrm{Mg}$ ha-1 (Carvalho et al., 2013; DE Aquino et al., 2018), mas pode atingir até $30 \mathrm{Mg}$ ha -1 ano -1 para safras de cana-de-açúcar de alto rendimento (Menandro et al., 2017).

Do ponto de vista agronômico, a prática de manter a palha da cana-de-açúcar na superfície do solo traz inúmeros serviços ecossistêmicos em longo prazo, incluindo menor variação na temperatura do solo, melhor infiltração e disponibilidade 
de água devido à menor evapotranspiração, controle eficaz de ervas daninhas e proteção contra a erosão do solo (Carvalho et al., 2017). Este último é um assunto muito importante nas lavouras de cana-de-açúcar. O solo coberto com palha de cana reduz a erosão do solo ao dissipar a energia cinética das gotas de chuva, diminuindo a velocidade do fluxo e aumentando a profundidade da camada de água na superfície do solo (Martins filho et al., 2009).

Ainda que a palhada sobre a superfície do solo possa beneficiar a sua qualidade a longo prazo e a produtividade da cultura, trazendo benefícios as propriedades do solo, esse resíduo também representa uma valiosa matéria-prima para a produção de etanol de segunda geração e cogeração de bioeletricidade e permite novas oportunidades para a indústria de canade-açúcar brasileira (Menandro et al., 2017). No entanto, apesar do alto potencial para uso industrial, faltam dados que informem a quantidade recomendável de palha que deve ser retirada do campo levando-se em consideração todos esses aspectos. A combinação equilibrada desses aspectos certamente ajudará a promover uma cadeia produtiva da cana-de-açúcar mais rentável e sustentável (Carvalho et al., 2017; Castioni et al., 2019).

A colheita mecanizada sem queima apresenta vantagens, em relação ao depósito de palhada no solo, tais como:

- Incrementos de matéria orgânica do solo (Pinheiro et al., 2010);

- $\quad$ Aumento na retenção de umidade do solo (Castioni et al., 2019; Pinheiro et al., 2010);

- Proteção contra erosão do solo (Brasil et al., 2016);

- $\quad$ Reciclagem de nutrientes da palhada (Menandro et al., 2017);

- Preservação da micro, meso e macrofauna do solo (Segnini et al., 2013).

O sistema de colheita sem queima é bem conhecido na literatura científica como uma estratégia promissora por seus benefícios envolvendo aspectos agronômicos e ambientais. No entanto, neste sistema de colheita, os elevados níveis de compactação do solo foram reconhecidos como o principal problema nos sistemas tradicionais de cultivo de cana-de-açúcar no Brasil, devido ao tráfego intenso durante a colheita mecanizada (Souza et al., 2014). Com a intensificação da mecanização nas lavouras de cana-de-açúcar, tanto no plantio, quanto na colheita, desde 2008, tem sido observada uma queda substancial na produtividade da cultura (Bordonal et al., 2018). As melhores práticas de manejo são e continuarão sendo cruciais para superar esse problema, incluindo a retenção da palha nos canaviais, a inclusão da rotação de culturas no ciclo da cana-de-açúcar e a adoção do plantio direto. Essas estratégias de manejo são essenciais para aumentar a produtividade da cana-de-açúcar.

\subsection{Manejo da Palhada e carbono orgânico do solo}

Desde o início dos anos 2000, o banimento gradual das queimadas antes da colheita da cana-de-açúcar aumentou a quantidade de palha deixada no solo. Após cada colheita de cana-de-açúcar, cerca de $15 \mathrm{Mg}$ ha -1 de palha, principalmente folhas e pequenos caules, são deixados na superfície do solo. Uma parte é necessária para a proteção do solo e manutenção do carbono orgânico do solo (SOC), mas este material também tem um alto potencial para produção de etanol de segunda geração ou bioeletricidade. Portanto, um dos principais desafios é quantificar a quantidade de palhada que pode ser removida de forma sustentável dos canaviais para a produção de energia sem comprometer o funcionamento do solo e outros serviços ecossistêmicos (Carvalho et al., 2017; Cherubin et al., 2018).

A decomposição da palhada da cana-de-açúcar é um processo de grande importância para indicar as orientações de manejo da palhada. A matéria orgânica do solo (MOS) desempenha múltiplas funções no ambiente como um todo, afeta direta ou indiretamente as propriedades químicas, físicas e biológicas do solo e é considerada um dos principais indicadores da qualidade do solo (Cherubin et al., 2016). De maneira geral, os estudos comparando sistema com e sem queima de palhada, destacam efeitos positivos sob eliminação do fogo perante a colheita e principalmente sobre as propriedades do solo (Ceddia et al., 1999; DOS Santos et al., 2020; Schultz et al., 2010). 
A decomposição da palhada no solo é uma fonte importante do pool de carbono orgânico do solo (SOC) (Wang et al., 2015), que também é uma etapa fundamental do ciclo do carbono no sistema planta-solo. A decomposição dos resíduos vegetais, fornece nutrientes importantes para o ciclo da cultura, facilita a retenção de água, transforma as propriedades físicas do solo e melhora a produtividade e a atividade dos organismos do solo (Yuan et al., 2021). Dessa forma, mudanças nos estoques de SOC resultam em modificações nos atributos físicos, químicos e biológicos do solo (Cherubin et al., 2016). Santos et al. (2020), relataram em estudo que a queima da cana-de-açúcar influencia e modifica quantitativamente e qualitativamente o conteúdo de SOC. Vários fatores afetam direta ou indiretamente a taxa de decomposição, sendo os mais importantes a quantidade e a qualidade da palha, as condições edafoclimáticas e as práticas de manejo (Sousa et al., 2017; G. Zhou et al., 2015)

Estudos mostraram que em clima tropical, como no caso do Brasil, onde a mineralogia de argila e o elevado grau de intemperismo resultam em baixa reserva de nutrientes, a preservação da palhada na colheita sem a queima contribui para a manutenção da fertilidade do solo podendo aumentá-la (Pinheiro et al., 2010). Assim, compreender a dinâmica do carbono orgânico do solo (SOC) é importante para a sustentabilidade de longo prazo dos agroecossistemas e do meio ambiente (Yuan et al., 2021).

O tempo de decomposição da palhada da cana pode variar de 12 a 14 meses, a depender da variedade utilizada e das condições edafoclimáticas as quais se encontram. Em regiões de clima tropical a taxa de decomposição pode ser até 10 vezes maior quando comparada com as regiões de clima temperado (Thorburn et al., 2012). Em um estudo, foi observado que a taxa de degradação da palhada é mais alta quanto maior a quantidade de palhada, e maior a umidade, o que contribui para uma decomposição mais acelerada e rápida (White \& Webber, 2018). Possivelmente esse efeito ocorre devido à palhada propiciar um microclima mais estável e favorável aos organismos que de maneira direta e/ou indireta participam da decomposição da palhada. A deposição da palhada na estação chuvosa induz uma decomposição mais acelerada, devido à maior umidade do solo e temperatura mais elevada (Pimentel et al., 2019; White \& Webber, 2018).

A permanência dos resíduos de qualquer cultura, faz com que o solo passe a acumular $\mathrm{C}$, decorrente do processo de decomposição da matéria orgânica do solo (MOS), ocorrendo a uma taxa de 0,73 Mg ha-1 ano-1 em solos arenosos e de 2,04 Mg ha-1 ano-1 para solos argilosos, ou a uma taxa média geral de 1,5 Mg C ha-1 ano-1 (Cerri et al., 2011). Assim, esse acúmulo do C no solo é dependente da entrada e das taxas de decomposição da matéria orgânica no solo (Zhou et al., 2019). Nesse sistema, o C da palhada da cana, é o principal responsável pela entrada de C orgânico no solo e também pela saída. A saída, ocorre pelo próprio processo de decomposição da palha e a respiração microbiana (Carmo et al., 2013; Carvalho et al., 2013)

Ao considerar a palha de cana-de-açúcar, que apresenta $47 \%$ de C (Yamaguchi et al., 2017), e que no sistema com colheita sem queima da palha são adicionados de 10 a 20 Mg ha-1 ano-1 de matéria seca sobre o solo (Schultz et al., 2010), têm-se um aporte de $\mathrm{C}$ anual que varia de 4,7 a $9,4 \mathrm{Mg}$ ha-1. Entretanto não se sabe ao certo quanto desse $\mathrm{C}$ vai ser efetivamente estabilizado no solo, e como a retirada de parte da palha da cana-de-açúcar pode influenciar nesse processo, já que isso também depende do potencial de armazenamento de carbono do solo, das condições climáticas e do tempo de instalação dos experimentos (Corbeels et al., 2016; Jiang et al., 2017; Thorburn et al., 2012; Zhao et al., 2016; Zhu et al., 2015).

A taxa de sequestro de $C$ apresenta resposta linear a deposição de palha (taxa de sequestro de $C=0,29 \mathrm{x}$ entrada anual de C - 0,57) (Jiang et al., 2017). A partir disso, fica claro que, nessa área, para garantir a manutenção do conteúdo atual de $C$ no solo deve-se adicionar no mínimo 1,89 Mg C ha-1 ano-1 (Jiang et al., 2017). Ou seja, o recolhimento de toda palha, diminuiria os níveis de $\mathrm{C}$ do solo. As maiores taxas de decomposição da palha ocorrem durante os primeiros seis meses após a colheita da cana-de-açúcar. Após esse período, a quantidade de palha na superfície do solo é reduzida significamente. Em áreas 
onde é feita a remoção de palhada para bioenergia ou álcool de $2^{\circ}$ geração, é importante que a colheita da cana-de-açúcar ocorra em uma época, em que o período de baixa cobertura de palha (6 a 12 meses) não coincida com a estação seca, quando a escassez de água pode comprometer o crescimento e o desenvolvimento da cultura (Pimentel et al., 2019). Em estudo avaliando a decomposição da palhada de cana-de-açúcar sob diferentes taxas de remoção da palha $(0 \% ; 25 \%$; 50\% e $100 \%$ da palhada removida). Pimentel (2019) e seus colaboradores, sugerem que a taxa de remoção mais adequada para manter a qualidade do solo e atender as demandas de produção de energia é de 50\% do total da palhada depositada no solo.

\subsection{Transformações dos resíduos orgânicos no solo}

Os restos vegetais que são depositados na superfície do solo, passam inicialmente, à transformação parcial pela macro e mesofauna e, posteriormente, à ação decompositora dos microrganismos. Parte do carbono presente nos resíduos é liberada para atmosfera como $\mathrm{CO} 2$, num processo denominado de mineralização, e o restante passa a fazer parte da MOS (Shnitzer \& Khan, 1972). A decomposição é resultado, principalmente, da despolimerização e oxidação de moléculas relativamente grandes, tais como as poliaromáticas, carboidratos, lipídios e proteínas, em moléculas menores e mais simples, como os ácidos carboxílicos, aminoácidos e CO2 (Silva \& Pasqual, 1999; Sollins et al., 1996).

A MOS é resultado, principalmente, da deposição de resíduos de plantas (fonte primária) e animais (fonte secundária) em vários estágios de decomposição, além dos organismos, vivos ou mortos, microrganismos e das raízes dos vegetais (Theng, 1989). Sua constituição é complexa, sendo formada por compostos ou frações com tempos de residência variando desde semanas até milhares de anos (Brady, 1989). Dessa forma, as substâncias orgânicas presentes no solo vão desde compostos lábeis, com elevada biodisponibilidade, até componentes quimicamente mais estáveis, em íntima associação com a fase mineral (Christensen, 1992).

O processo de humificação da MOS compreende a transformação dos compostos orgânicos com morfologia conhecida e identificável em compostos húmicos, que são as substâncias húmicas de elevado peso molecular e alta estabilidade (Piccolo et al., 2018). A estabilização significa o decréscimo do potencial de perda por oxidação, erosão ou lixiviação (Sollins et al., 1996). A estabilidade da MOS e do carbono orgânico é resultante de três conjuntos gerais de características: recalcitrância, interações e acessibilidade, a qual por definição aumenta com a recalcitrância e decresce com a acessibilidade (Sollins et al., 1996).

A sua estabilidade é atribuída à estrutura química e heterogeneidade, assim como captura dentro dos agregados do solo e principalmente devido à interação com cátions metálicos e minerais de argila (Freixo et al., 2002). Em função do compartimento onde se encontra as frações da MOS, a velocidade de decomposição pode variar, podendo ser menor para o material orgânico que se encontra exposto (desprotegido) no solo e maior se estiver protegido, por exemplo dentro de agregados (Freixo et al., 2002).

\subsubsection{Matéria orgânica do solo e a estabilização das Substâncias húmicas}

O processo de estabilização da MO do solo começa com a formação de ácidos fúlvicos e segue em direção a ácidos húmicos e depois humina (Rosa et al., 2017). A maior parte da MOS é formada pelas substâncias húmicas (SH) definidas como a fração recalcitrante, sua formação é caracterizada por um processo complexo baseado na síntese e, ou, ressíntese dos produtos da mineralização dos compostos orgânicos que chegam ao solo (Baldotto \& Baldotto, 2014; Han et al., 2016). Constituem a maior parte do carbono orgânico total (COT) nos solos, de 60 a 80\%, apresentando alta reatividade e interação com as superfícies minerais e espécies químicas em solução (Piccolo et al., 2018). A alta reatividade é explicada pelo enriquecimento em grupos funcionais contendo oxigênio na forma de carboxilas, hidroxilas fenólicas e carbonilas (Piccolo, 2002). (Stevenson, 1994). 
De acordo com a Sociedade Internacional de Substâncias Húmicas (IHSS - International Humic Substance Society), as substâncias são classificadas de acordo com a solubilidade em diferentes valores de $\mathrm{pH}$ : ácidos fúlvicos (AF), quando solúveis em meio alcalino e ácido; ácidos húmicos (AH), quando solúveis em solução alcalina e insolúveis em meio ácido e huminas $(\mathrm{H})$, insolúveis em qualquer valor de pH. Com relação às características químicas das substâncias húmicas, destacamse:

(i) Ácido Fúlvico - Fração colorida que se mantém solúvel em meio alcalino ou em meio ácido diluído. Quimicamente são constituídos, sobretudo, por polissacarídeos, aminoácidos, compostos fenólicos, etc. Apresentam um alto conteúdo de grupos carboxílicos e seu peso molecular é relativamente baixo. Combinam-se com óxidos de $\mathrm{Fe}, \mathrm{Al}$, argilas e outros compostos orgânicos. Possuem propriedades redutoras e formam complexos estáveis com $\mathrm{Fe}, \mathrm{Cu}, \mathrm{Ca}$ e $\mathrm{Mg}$ (Stevenson, 1994).

(ii) Ácidos Húmicos - Fração escura solúvel em meio alcalino, precipitando-se em forma de produto escuro e amorfo em meio ácido. Quimicamente são muito complexos, formados por polímeros compostos aromáticos e alifáticos com elevado peso molecular, e grande capacidade de troca catiônica. Combina-se com elementos metálicos formando humatos, que podem precipitar (humatos de cálcio, magnésio, etc.) ou permanecer em dispersão coloidal (humatos de sódio, potássio, amônio, etc.) (Stevenson, 1994).

(iii) Humina - Fração insolúvel em meio alcalino ou em meio ácido diluído. Possui reduzida capacidade de reação. É a fração mais antiga e estável da MOS (Stevenson, 1994).

Estruturalmente, as três frações húmicas são similares, mas diferem em peso molecular e conteúdo de grupos funcionais, como apresentado no quadro 1. O carbono das frações AH e HUM, são mais estáveis, em detrimento do carbono da fração AF, que é mais solúvel e apresenta maior mobilidade no solo (Sousa et al., 2020). Nesse contexto os AF possuem o menor peso molecular, menos carbono e nitrogênio e tem o mais alto conteúdo de grupos funcionais oxigenados $(\mathrm{CO} 2 \mathrm{H}, \mathrm{OH}$, $\mathrm{C}=\mathrm{O}$ ) por unidade de peso que as demais frações húmicas. A estrutura química e propriedades da fração HUM parecem ser similares àquelas dos $\mathrm{AH}$. A insolubilidade da HUM pode ser proveniente dela ter sido firmemente adsorvida a constituintes inorgânicos do solo. A resistência à degradação microbiana dos materiais húmicos parece também ser em grande parte devido à formação de complexos metálicos e/ou argilo-orgânicos estáveis (Schnitzer,1978).

Os ácidos húmicos possuem maior conteúdo de $\mathrm{C}$ e menor de $\mathrm{O}$, e uma massa maior que os ácidos fúlvicos. Com o grau de polimerização relativamente maior dos ácidos húmicos, é possível constatar um estágio mais avançado de humificação. Entretanto, os ácidos fúlvicos possuem mais agrupamentos carboxilas $(\mathrm{COOH})$ por unidade de massa em relação aos ácidos húmicos que somados com os grupamentos fenólicos, caracterizam uma maior acidez total, apresentando maior capacidade de troca catiônica (CTC) que os ácidos húmicos (Benites et al., 2003).

Quadro 1. Propriedades químicas das substâncias húmicas do solo.

\begin{tabular}{|c|c|c|c|}
\hline Propriedades químicas & Ácidos fúlvicos & Ácidos húmicos & Humina \\
\hline Peso molecular (D) & $640-5000$ & $10000-100000$ & $>100000$ \\
\hline $\mathrm{C}(\%)$ & $42-47$ & $51-62$ & $>62$ \\
\hline $\mathrm{O}(\%)$ & $45-50$ & $31-36$ & $>30$ \\
\hline $\mathrm{N}(\%)$ & $2,0-4,1$ & $3,6-5,5$ & $<5$ \\
\hline $\mathrm{CTC}\left(\mathrm{cmolc} \mathrm{kg}^{-1}\right)$ & $\sim 1400$ & $\sim 500$ & $<0$ \\
\hline
\end{tabular}

Fonte: Adaptado de (Benites et al., 2003). 


\subsubsection{Importância e interferência do manejo da palhada de cana-de-açúcar sobre as substâncias húmicas}

As substâncias húmicas, contribuem de maneira significativa, com a melhoria das propriedades físicas, químicas e biológicas do solo. Os benefícios da alteração do solo com as SH incluem agregação com maior estabilidade, aumento da capacidade de tamponamento do $\mathrm{pH}$ e troca de cátions, maior capacidade de retenção de água, maior biodisponibilidade de nutrientes imóveis (como P, Fe e Zn) e diminuição da toxicidade do alumínio e metais pesados (Chen et al., 2004; Piccolo, 2002). Além de influenciar indiretamente a produtividade da planta através de modificações das características do solo, as SH também podem impactar diretamente nos processos metabólicos da planta, como bem relatado por diversos autores (Aguiar et al., 2018; Canellas et al., 2015; Canellas \& Olivares, 2014; Canellas \& Façanha, 2004; Canellas et al., 2019; Chen et al., 2004; De Hita et al., 2020; García et al., 2019; García et al., 2016; Olivares et al., 2017; Nardi et al., 2000, 2009).

Mudanças iniciais são esperadas na fração AF, já que reflete o primeiro estádio em direção à estabilização da matéria orgânica (Rosa et al., 2017). Em sistemas de colheita com a queima prévia do canavial, as frações AF, AH e HUM são afetadas pela duração do manejo da queima. A fração HUM é a menos impactada, apresentando carbono humificado estável com alto grau de condensação e interação com a matriz mineral do solo. A queima de longa duração afetou principalmente as frações mais lábeis (AF) da MOS (Santos et al., 2020). A fração AF é composta predominantemente por estruturas altamente funcionalizadas com baixa aromaticidade. As frações mais recalcitrantes de SOM, como AH e HUM, eram mais resistentes à queima. (Benites et al., 2003; García et al., 2016).

A remoção excessiva da palha esgota os reservatórios de matéria orgânica do solo. Segundo Morais et al., 2020), a retirada de $50 \%$ da palhada da superfície do solo, não afeta os índices de qualidade da matéria orgânica do solo, não interferindo nas frações húmicas. Silva et al., 2020, também observou alterações nas frações húmicas (AH e HUM) em áreas onde houve a retirada de toda a palhada da superfície do solo, enquanto, no manejo de 50\% da palhada e $100 \%$ sobre a superfície do solo, foi suficiente para a preservação e/ou manutenção das frações AH e HUM. A fração AF não obteve resposta nesse estudo à remoção da palhada.

\section{Considerações Finais}

A MOS é resultado, principalmente, da deposição de resíduos de plantas e animais em vários estágios de decomposição, além dos organismos, vivos ou mortos, microrganismos e das raízes dos vegetais. As substâncias húmicas ( $\mathrm{SH}$ ) é um indicativo da qualidade da matéria orgânica e do manejo do solo. A remoção de toda a palhada do solo deve ser avaliada com cautela, a fim de preservar as propriedades do solo, uma vez que as SH estão associadas as propriedades físicas, químicas e biológicas do solo. Isto irá permitir atos de gestão que levem a preservar os seus efeitos benéficos e, ao mesmo tempo, contemplar a fabricação de álcool de segunda geração e a co-geração de energia.

Novos estudos acerca do manejo do solo, e respectivas tecnologias implementadas para a otimização da produção sulcroalcooleira e suas interações com a qualidade do solo serão imprescindíveis para que seja resguardada a sustentabilidade dessa atividade, dada a necessidade atual de buscar soluções inovadoras para produção agrícola aperfeiçoada em uma gestão eficiente dos recursos naturais. Garantir a qualidade do solo é imprescindível para continuarmos produzindo alimentos e usando a biomassa produzida na geração de bioenergia.

\section{Referências}

Aguiar, N. O., Olivares, F. L., Novotny, E. H., \& Canellas, L. P. (2018). Changes in metabolic profiling of sugarcane leaves induced by endophytic diazotrophic bacteria and humic acids. PeerJ, 2018(9), 1-28. https://doi.org/10.7717/peerj.5445

Baldotto, M. A., \& Baldotto, L. E. B. (2014). Ácidos Húmicos. Revista Ceres, 61, 856-881. https://doi.org/10.1590/0034-737X201461000011 
Benites, V. M., Madari, B., \& Luiz de A Machado, P. O. (2003). Extração e Fracionamento Quantitativo de Substâncias Húmicas do Solo: um Procedimento Simplificado de Baixo Custo Técnico Comunicado Introdução.

Bordonal, R. de O., Carvalho, J. L. N., Lal, R., de Figueiredo, E. B., de Oliveira, B. G., \& La Scala, N. (2018). Sustainability of sugarcane production in Brazil. A review. In Agronomy for Sustainable Development (Vol. 38, Issue 2, pp. 1-23). Springer-Verlag France. https://doi.org/10.1007/s13593-018-0490-x

Brasil, C., Valim, S., Pavei, A., Sobrinho, T., \& Almeida, S. (2016). Semina: Ciências Agrárias. Semina: Ciências Agrárias, 37(3), 1155-1164. https://doi.org/10.5433/1679-0359.2016v37n3p1155

Canellas, L., \& Santos, G. (2005). Humosfera: tratado preliminar sobre a química das substâncias húmicas.

Canellas, Luciano P., \& Olivares, F. L. (2014). Physiological responses to humic substances as plant growth promoter. Chemical and Biological Technologies in Agriculture, 1(1), 1-11. https://doi.org/10.1186/2196-5641-1-3

Canellas, Luciano P., Olivares, F. L., Aguiar, N. O., Jones, D. L., Nebbioso, A., Mazzei, P., \& Piccolo, A. (2015). Humic and fulvic acids as biostimulants in horticulture. In Scientia Horticulturae (Vol. 196, pp. 15-27). Elsevier. https://doi.org/10.1016/j.scienta.2015.09.013

Canellas, Luciano Pasqualoto, \& Façanha, A. R. (2004). Chemical nature of soil humified fractions and their bioactivity. Pesquisa Agropecuaria Brasileira, 39(3), 233-240. https://doi.org/10.1590/s0100-204x2004000300005

Canellas, N. O. A., Olivares, F. L., \& Canellas, L. P. (2019). Metabolite fingerprints of maize and sugarcane seedlings: searching for markers after inoculation with plant growth-promoting bacteria in humic acids. Chem. Biol. Technol. Agric, 6, 14. https://doi.org/10.1186/s40538-019-0153-4

Cardoso, T. F., Watanabe, M. D. B., Souza, A., Chagas, M. F., Cavalett, O., Morais, E. R., Nogueira, L. A. H., Leal, M. R. L. V., Braunbeck, O. A., Cortez, L. A. B., \& Bonomi, A. (2019). A regional approach to determine economic, environmental and social impacts of different sugarcane production systems in Brazil. Biomass and Bioenergy, 120, 9-20. https://doi.org/10.1016/j.biombioe.2018.10.018

Carmo, J. B. do, Filoso, S., Zotelli, L. C., De Sousa Neto, E. R., Pitombo, L. M., Duarte-Neto, P. J., Vargas, V. P., Andrade, C. A., Gava, G. J. C., Rossetto, R., Cantarella, H., Neto, A. E., \& Martinelli, L. A. (2013). Infield greenhouse gas emissions from sugarcane soils in brazil: Effects from synthetic and organic fertilizer application and crop trash accumulation. GCB Bioenergy, 5(3), 267-280. https://doi.org/10.1111/j.1757-1707.2012.01199.x

Carvalho, Joao L.N., Hudiburg, T. W., Franco, H. C. J., \& DeLucia, E. H. (2017). Contribution of above- and belowground bioenergy crop residues to soil carbon. GCB Bioenergy, 9(8), 1333-1343. https://doi.org/10.1111/gcbb.12411

Carvalho, João Luís Nunes, Nogueirol, R. C., Menandro, L. M. S., Bordonal, R. de O., Borges, C. D., Cantarella, H., \& Franco, H. C. J. (2017). Agronomic and environmental implications of sugarcane straw removal: a major review. In GCB Bioenergy (Vol. 9, Issue 7, pp. 1181-1195). Blackwell Publishing Ltd. https://doi.org/10.1111/gcbb.12410

Carvalho, João Luís Nunes, Otto, R., Franco, H. C. J., \& Trivelin, P. C. O. (2013). Input of sugarcane post-harvest residues into the soil. Scientia Agricola, 70(5), 336-344. https://doi.org/10.1590/S0103-90162013000500008

Castioni, G. A. F., Cherubin, M. R., Bordonal, R. de O., Barbosa, L. C., Menandro, L. M. S., \& Carvalho, J. L. N. (2019). Straw Removal Affects Soil Physical Quality and Sugarcane Yield in Brazil. Bioenergy Research, 12(4), 789-800. https://doi.org/10.1007/s12155-019-10000-1

Castro, S. G. de Q., Dinardo-Miranda, L. L., Fracasso, J. V., Bordonal, R. O., Menandro, L. M. S., Franco, H. C. J., \& Carvalho, J. L. N. (2019). Changes in Soil Pest Populations Caused by Sugarcane Straw Removal in Brazil. Bioenergy Research, 12(4), 878-887. https://doi.org/10.1007/s12155-019-10019-4

Ceddia, M. B., Dos Anjos, L. H. C., Lima, E., Neto, A. R., \& Da Silva, L. (1999). Sugar cane harvesting systems and changes on physical properties of a yellow podzolic soil in Espírito Santo, Brazil. Pesquisa Agropecuaria Brasileira, 34(8), 1467-1473. https://doi.org/10.1590/s0100-204x1999000800019

Cerri, C. C., Galdos, M. V., Maia, S. M. F., Bernoux, M., Feigl, B. J., Powlson, D., \& Cerri, C. E. P. (2011). Effect of sugarcane harvesting systems on soil carbon stocks in Brazil: An examination of existing data. European Journal of Soil Science, 62(1), 23-28. https://doi.org/10.1111/j.1365-2389.2010.01315.x

Chen, Y., Magen, H., \& Clapp, C. E. (2004). Mechanisms of plant growth stimulation by humic substances: The role of organo-iron complexes. Soil Science and Plant Nutrition, 50(7), 1089-1095. https://doi.org/10.1080/00380768.2004.10408579

Cherubin, Maurício R., Karlen, D. L., Franco, A. L. C., Cerri, C. E. P., Tormena, C. A., \& Cerri, C. C. (2016). A Soil Management Assessment Framework (SMAF) Evaluation of Brazilian Sugarcane Expansion on Soil Quality. Soil Science Society of America Journal, 80(1), 215-226. https://doi.org/10.2136/sssaj2015.09.0328

Cherubin, Maurício Roberto, Oliveira, D. M. D. S., Feigl, B. J., Pimentel, L. G., Lisboa, I. P., Gmach, M. R., Varanda, L. L., Morais, M. C., Satiro, L. S., Popin, G. V., De Paiva, S. R., Dos Santos, A. K. B., De Vasconcelos, A. L. S., De Melo, P. L. A., Cerri, C. E. P., \& Cerri, C. C. (2018). Crop residue harvest for bioenergy production and its implications on soil functioning and plant growth: A review. Scientia Agricola, 75(3), 255-272. https://doi.org/10.1590/1678992x-2016-0459

CHRISTENSEN, B. T. (1992). Physical fractionation of soil and organic matter in primary particle size and density separates. Adv. Soil Science, $20,1-90$. Corbeels, M., Marchão, R. L., Neto, M. S., Ferreira, E. G., Madari, B. E., Scopel, E., \& Brito, O. R. (2016). Evidence of limited carbon sequestration in soils under no-tillage systems in the Cerrado of Brazil. Scientific Reports, 6(1), 1-8. https://doi.org/10.1038/srep21450

da Silva, M. J., Lucas, L. de, Correa, M. H. F., \& de Souza, C. H. W. (2021). Quality Indexes and Performance in Mechanized Harvesting of Sugarcane at a Burnt Cane and Green Cane. Sugar Tech, 1-9. https://doi.org/10.1007/s12355-021-00957-9

de Aquino, G. S., de Conti Medina, C., Shahab, M., Santiago, A. D., Cunha, A. C. B., Kussaba, D. A. O., Carvalho, J. B., \& Moreira, A. (2018). Does straw mulch partial-removal from soil interfere in yield and industrial quality sugarcane? A long term study. Industrial Crops and Products, 111, 573-578. https://doi.org/10.1016/j.indcrop.2017.11.026

De Beni Arrigoni, E. (2013). Main pests of the new system. Revista Opiniões. 
De Hita, D., Fuentes, M., Fernández, V., Zamarreño, A. M., Olaetxea, M., \& García-Mina, J. M. (2020). Discriminating the Short-Term Action of Root and Foliar Application of Humic Acids on Plant Growth: Emerging Role of Jasmonic Acid. Frontiers in Plant Science, 11(April), 1-14. https://doi.org/10.3389/fpls.2020.00493

de Vasconcelos, R. F. B., de Souza, E. R., Cantalice, J. R. B., \& Silva, L. S. (2014). Physical quality of yellow oxisol of a coastal plain under different management systems in sugarcane. Revista Brasileira de Engenharia Agricola e Ambiental, 18(4), 381-386. https://doi.org/10.1590/S141543662014000400004

Dinardo-Miranda, L. L., \& Fracasso, J. V. (2013). Sugarcane straw and the populations of pests and nematodes. In Scientia Agricola. 70(5), 369-374. Scientia Agricola. https://doi.org/10.1590/s0103-90162013000500012

dos Santos, O. A. Q., Tavares, O. C. H., García, A. C., Rossi, C. Q., de Moura, O. V. T., Pereira, W., da Silva Rodrigues Pinto, L. A., Berbara, R. L. L., \& Pereira, M. G. (2020). Fire lead to disturbance on organic carbon under sugarcane cultivation but is recovered by amendment with vinasse. Science of the Total Environment, 739, 140063. https://doi.org/10.1016/j.scitotenv.2020.140063

Du, C., Kulay, L., Cavalett, O., Dias, L., \& Freire, F. (2018). Life cycle assessment addressing health effects of particulate matter of mechanical versus manual sugarcane harvesting in Brazil. International Journal of Life Cycle Assessment, 23(4), 787-799. https://doi.org/10.1007/s11367-017-1334-7

FAO. (2015). Organização das Nações Unidas para Agricultura e Alimentação: Representante da FAO Brasil apresenta cenário da demanda por alimentos | FAO no Brasil | Food and Agriculture Organization of the United Nations. http://www.fao.org/brasil/noticias/detail-events/en/c/901168/

\section{FAO. (2020). OCDE-FAO Perspectivas Agrícolas 2020-2029. https://doi.org/10.1787/a0848ac0-es}

Frank J. Stevenson. (1994). Humus Chemistry: Genesis, Composition, Reactions - F. J. Stevenson - Google Livros. https://books.google.com.br/books?hl=ptBR\&lr=\&id=7kCQch_YKoMC\&oi=fnd\&pg=PA1\&ots=LJrVg55Vzq\&sig=a3_I-DqknIvpoFqUz4DEHq_HtjI\&redir_esc=y\#v=onepage\&q\&f=false

Freixo, A. A.; Canellas, L.P.; Machado, P. L. O. A. (2002). Propriedades espectrais da matéria orgânica leve-livre e leve intra-agregado de dois Latossolos sob plantio direto e preparo convencional. Revista Brasileira de Ciências Do Solo, 26, 445-453.

García, Andrés C., van Tol de Castro, T. A., Berbara, R. L. L., Tavares, O. C. H., Elias, S. S., de Amaral Sobrinho, N. M. B., Pereira, M. G., \& Zonta, E. (2019). Critical review about structure-functions relationship for humic substances interactions with plant oxidative metabolism. Revista Virtual de Quimica, 11(3), 754-770. https://doi.org/10.21577/1984-6835.20190055

García, Andrés Calderín, Olaetxea, M., Santos, L. A., Mora, V., Baigorri, R., Fuentes, M., Zamarreño, A. M., Berbara, R. L. L., \& Garcia-Mina, J. M. (2016). Involvement of Hormone-and ROS-Signaling Pathways in the Beneficial Action of Humic Substances on Plants Growing under Normal and Stressing Conditions. BioMed Research International, 2016. https://doi.org/10.1155/2016/3747501

García, Andrés Calderín, Santos, L. A., de Souza, L. G. A., Tavares, O. C. H., Zonta, E., Gomes, E. T. M., García-Mina, J. M., \& Berbara, R. L. L. (2016). Vermicompost humic acids modulate the accumulation and metabolism of ROS in rice plants. Journal of Plant Physiology, 192, 56-63. https://doi.org/10.1016/j.jplph.2016.01.008

Han, L., Sun, K., Jin, J., \& Xing, B. (2016). Some concepts of soil organic carbon characteristics and mineral interaction from a review of literature. In Soil Biology and Biochemistry (Vol. 94, pp. 107-121). Elsevier Ltd. https://doi.org/10.1016/j.soilbio.2015.11.023

IHSS. (n.d.). O que são substâncias húmicas | IHSS. Retrieved May 14, 2021, from https://humic-substances.org/what-are-humic-substances-2/

Jiang, C. M., Yu, W. T., Ma, Q., Xu, Y. G., \& Zou, H. (2017). Alleviating global warming potential by soil carbon sequestration: A multi-level straw incorporation experiment from a maize cropping system in Northeast China. Soil and Tillage Research, 170, 77-84. https://doi.org/10.1016/j.still.2017.03.003

Lopes, I. M., Pinheiro, É. F. M., Lima, E., Ceddia, M. B., De Campos, D. V. B., \& Alves, B. J. (2017). Emissions of N2O in soils under sugarcane cultivation in the atlantic forest biome: Effect of harvesting systems and vinasse fertilization. Revista Virtual de Quimica, 9(5), 1930-1943. https://doi.org/10.21577/19846835.20170113

Lopes Olivares, F., Galba Busato, J., De Paula, A. M., Da, L., Lima, S., Oliveira Aguiar, N., \& Canellas, L. P. (2017). Plant growth promoting bacteria and humic substances: crop promotion and mechanisms of action Open Access. Chem. Biol. Technol. Agric, 4, 30. https://doi.org/10.1186/s40538-017-0112-x

Macedo, N., \& Macedo, D. (2004). As pragas de maior incidência nos canaviais e seus controles.

Martins Filho, M. V., Liccioti, T. T., Pereira, G. T., Marques, J. M., \& Sanchez, R. B. (2009). Perdas de solo e nutrientes por erosão num Argissolo com resíduos vegetais de cana-de-açúcar. Engenharia Agricola, 29(1), 8-18. https://doi.org/10.1590/S0100-69162009000100002

Menandro, L. M. S., Cantarella, H., Franco, H. C. J., Kölln, O. T., Pimenta, M. T. B., Sanches, G. M., Rabelo, S. C., \& Carvalho, J. L. N. (2017). Comprehensive assessment of sugarcane straw: implications for biomass and bioenergy production. Biofuels, Bioproducts and Biorefining, 11(3), 488-504. https://doi.org/10.1002/bbb.1760

Nardi, S., Carletti, P., Pizzeghello, D., \& Muscolo, A. (2009). Biological Activities of Humic Substances. Biophysico-Chemical Processes Involving Natural Nonliving Organic Matter in Environmental Systems, 305-339. https://doi.org/10.1002/9780470494950.ch8

Nardi, S., Pizzeghello, D., Reniero, F., \& Rascio, N. (2000). Chemical and Biochemical Properties of Humic Substances Isolated from Forest Soils and Plant Growth. Soil Science Society of America Journal, 64(2), 639-645. https://doi.org/10.2136/sssaj2000.642639x

Paraiso, M. L. de S., \& Gouveia, N. (2015). Riscos à saúde devido à queima prévia da palha de cana-de-açúcar no Estado de São Paulo, Brasil. Revista Brasileira de Epidemiologia, 18(3), 691. https://doi.org/10.1590/1980-5497201500030014

Piccolo, A. (2002). The supramolecular structure of humic substances: A novel understanding of humus chemistry and implications in soil science. In Advances in Agronomy (Vol. 75, pp. 57-134). Academic Press Inc. https://doi.org/10.1016/s0065-2113(02)75003-7 
Pimentel, L. G., Cherubin, M. R., Oliveira, D. M. S., Cerri, C. E. P., \& Cerri, C. C. (2019). Decomposition of sugarcane straw: Basis for management decisions for bioenergy production. Biomass and Bioenergy, 122, 133-144. https://doi.org/10.1016/j.biombioe.2019.01.027

Pinheiro, É. F. M., Lima, E., Ceddia, M. B., Urquiaga, S., Alves, B. J. R., \& Boddey, R. M. (2010). Impact of pre-harvest burning versus trash conservation on soil carbon and nitrogen stocks on a sugarcane plantation in the Brazilian Atlantic forest region. Plant and Soil, 333(1), 71-80. https://doi.org/10.1007/s11104010-0320-7

Rosa, D. M., Nóbrega, L. H. P., Mauli, M. M., de Lima, G. P., \& Pacheco, F. P. (2017). Substâncias húmicas do solo cultivado com plantas de cobertura em rotação com milho e soja. Revista Ciencia Agronomica, 48(2), 221-230. https://doi.org/10.5935/1806-6690.20170026

Rossato, J. A. d. S., Costa, G. H. G., Madaleno, L. L., Mutton, M. J. R., Higley, L. G., \& Fernandes, O. A. (2013). Characterization and impact of the sugarcane borer on sugarcane yield and quality. Agronomy Journal, 105(3), 643-648. https://doi.org/10.2134/agronj2012.0309

Rossi, Celeste Q., Pereira, M. G., García, A. C., Perin, A., Gazolla, P. R., \& González, A. P. (2013). Fósforo em cronossequência de cana-de-açúcar queimada no cerrado goiano -Análise de ácidos húmicos por RMN de 31P. Quimica Nova, 36(8), 1126-1130. https://doi.org/10.1590/S0100-40422013000800009

Rossi, Celeste Queiroz, Pereira, M. G., García, A. C., Berbara, R. L. L., Gazolla, P. R., Perin, A., \& González, A. P. (2016). Effects on the composition and structural properties of the humified organic matter of soil in sugarcane strawburning: A chronosequence study in the Brazilian Cerrado of Goiás State. Agriculture, Ecosystems and Environment, 216, 34-43. https://doi.org/10.1016/j.agee.2015.09.022

Pereira, AS, Shitsuka, DM, Parreira, FJ \& Shitsuka, R. (2018). Metodologia da pesquisa científica. UFSM. https://repositorio.ufsm.br/bitstream/handle/1/15824/Lic_Computacao_Metodologia-Pesquisa-Cientifica.pdf?sequence=1 .

Schultz, N., Lima, E., Pereira, M. G., \& Zonta, E. (2010). Efeito residual da adubação na cana-planta e da adubação nitrogenada e potássica na cana-soca colhidas com e sem a queima da palhada. Revista Brasileira de Ciência Do Solo, 34(3), 811-820. https://doi.org/10.1590/s0100-06832010000300023

Segnini, A., Carvalho, J. L. N., Bolonhezi, D., Milori, D. M. B. P., da Silva, W. T. L., Simões, M. L., Cantarella, H., de Maria, I. C., \& Martin-Neto, L. (2013). Carbon stock and humification index of organic matter affected by sugarcane straw and soil management. Scientia Agricola, 70(5), 321-326. https://doi.org/10.1590/S0103-90162013000500006

Silva, L.M.V. \& Pasqual, A. (1999). Dinâmica e modelagem da matéria orgânica do solo com ênfase ao ecossistema tropical. Energia Na Agricultura, 14, 1324.

Sollins, P.; Homann, P.; Caldwell, B. A. (1996). Stabilization of soil organic matter: mechanisms and controls. Geoderma, 74, 65-105.

Sousa, M. A. de, Paz, V. R., Reis, I. M. S., Almada, A. P. de, Rossi, C. Q., Pereira, M. G., \& Pinto, L. A. R. da S. (2020). Brazilian Journal of Development Atributos químicos e frações da matéria orgânica em solos antrópicos na Amazônia Oriental Chemical attributes and fractions of organic matter in anthropic soils in the Eastern Amazon. Brazilian Journal of Development, 6(5), 29623-29643. https://doi.org/10.34117/bjdv6n5-424

Sousa, J. G. D. A., Cherubin, M. R., Cerri, C. E. P., Cerri, C. C., \& Feigl, B. J. (2017). Sugar cane straw left in the field during harvest: Decomposition dynamics and composition changes. Soil Research, 55(8), 758-768. https://doi.org/10.1071/SR16310

Souza, G. S. de, de Souza, Z. M., da Silva, R. B., Barbosa, R. S., \& Araújo, F. S. (2014). Controle de tráfego e seu efeito na qualidade física do solo e no cultivo da cana-de-açúcar. Revista Brasileira de Ciencia Do Solo, 38(1), 135-146. https://doi.org/10.1590/S0100-06832014000100013

Souza, R. A., Telles, T. S., Machado, W., Hungria, M., Filho, J. T., \& Guimarães, M. de F. (2012). Effects of sugarcane harvesting with burning on the chemical and microbiological properties of the soil. Agriculture, Ecosystems and Environment, 155, 1-6. https://doi.org/10.1016/j.agee.2012.03.012

Stevenson, F. J. (1994). Humus Chemistry: Genesis, Composition, Reactions.

Theng, B. K. G. et al. (1989). Clay-humic interactions and soil aggregate stability. (G. Coleman, D.C.; Oades, J.M.; Uehara (ed.)).

Thorburn, P. J., Meier, E. A., Collins, K., \& Robertson, F. A. (2012). Changes in soil carbon sequestration, fractionation and soil fertility in response to sugarcane residue retention are site-specific. Soil and Tillage Research, 120, 99-111. https://doi.org/10.1016/j.still.2011.11.009

Wang, W., Lai, D. Y. F., Wang, C., Pan, T., \& Zeng, C. (2015). Effects of rice straw incorporation on active soil organic carbon pools in a subtropical paddy field. Soil and Tillage Research, 152, 8-16. https://doi.org/10.1016/j.still.2015.03.011

White, P. M., \& Webber, C. L. (2018). Green-Cane Harvested Sugarcane Crop Residue Decomposition as a Function of Temperature, Soil Moisture, and Particle Size. Sugar Tech, 20(5), 497-508. https://doi.org/10.1007/s12355-017-0579-6

Yamaguchi, C. S., Ramos, N. P., Carvalho, C. S., Pires, A. M. M., \& de Andrade, C. A. (2017). Decomposição da palha de cana-de-açúcar e balanço de carbono em função da massa inicialmente aportada sobre o solo e da aplicação de vinhaça. Bragantia, 76(1), 135-144. https://doi.org/10.1590/1678-4499.580

Yuan, G., Huan, W., Song, H., Lu, D., Chen, X., Wang, H., \& Zhou, J. (2021). Effects of straw incorporation and potassium fertilizer on crop yields, soil organic carbon, and active carbon in the rice-wheat system. Soil and Tillage Research, 209, 104958. https://doi.org/10.1016/j.still.2021.104958

Zhao, S., Li, K., Zhou, W., Qiu, S., Huang, S., \& He, P. (2016). Changes in soil microbial community, enzyme activities and organic matter fractions under long-term straw return in north-central China. "Agriculture, Ecosystems and Environment," 216, 82-88. https://doi.org/10.1016/j.agee.2015.09.028

Zhou, G., Zhang, J., Mao, J., Zhang, C., Chen, L., Xin, X., \& Zhao, B. (2015). Mass loss and chemical structures of wheat and maize straws in response to ultraviolet-B radiation and soil contact. Scientific Reports, 5(1), 1-11. https://doi.org/10.1038/srep14851

Zhou, Z., Zeng, X., Chen, K., Li, Z., Guo, S., Shangguan, Y., Yu, H., Tu, S., \& Qin, Y. (2019). Long-term straw mulch effects on crop yields and soil organic carbon fractions at different depths under a no-till system on the Chengdu Plain, China. Journal of Soils and Sediments, 19(5), 2143-2152. https://doi.org/10.1007/s11368-018-02234-x

Zhu, L., Hu, N., Zhang, Z., Xu, J., Tao, B., \& Meng, Y. (2015). Short-term responses of soil organic carbon and carbon pool management index to different annual straw return rates in a rice-wheat cropping system. Catena, 135, 283-289. https://doi.org/10.1016/j.catena.2015.08.008 